\title{
A FTIR microspectroscopic study of the uptake and metabolism of isotopically labelled fatty acids by metastatic prostate cancer
}

\author{
E. Gazi ${ }^{\text {a,* }}$, T.J. Harvey ${ }^{\text {b }}$, M.D. Brown ${ }^{\text {a }}$, N.P. Lockyer $^{\text {b }}$, P. Gardner ${ }^{\text {b }}$, N.W. Clarke ${ }^{\text {a,c }}$ \\ ${ }^{a}$ Genito Urinary Cancer Research Group, School of Cancer and Imaging Sciences, Paterson Institute for Cancer Research, University of Manchester, \\ Wilmslow Road, Manchester M20 4BX, United Kingdom \\ ${ }^{\mathrm{b}}$ School of Chemical Engineering and Analytical Science, The University of Manchester, Manchester Interdisciplinary Biocentre, \\ 131 Princess Street, Manchester M1 7ND, United Kingdom \\ ${ }^{\mathrm{c}}$ Department of Urology, Hope Hospital NHS Trust, Manchester M6 8HD, United Kingdom
}

\section{A R T I C L E I N F O}

Article history:

Received 16 January 2008

Received in revised form 18 August 2008

Accepted 15 September 2008

Available online 2 October 2008

Keywords:

Infrared microspectroscopy

Palmitic acid

Arachidonic acid

Deuterated fatty acids

Metabolomics

Prostate cancer

\begin{abstract}
A B S T R A C T
Recent epidemiological studies have positively associated total dietary fat intake to prostate cancer (CaP) incidence and progression. However, the role of fat-specific intake remains unclear through these patient-orientated studies, which in-turn warrants the need for controlled molecular based investigations. FTIR microspectroscopy is now a well established tool for the metabolomic analysis of a wide variety of biomolecules at the whole cell level and can be used to generate hypotheses to refine molecular specific experiments. For the first time, we have used FTIR microspectroscopy to measure the uptake and metabolism of the saturated fatty acid (FA), palmitic acid (PA) and the unsaturated FA, arachidonic acid (AA) using deuterated analogues. We also report on the temporal fluctuations of different biomolecular domains (lipid, phosphate and protein secondary structure) within PC-3 cells in response to $D_{8}-A A$ and $D_{31}-P A$ uptake. Our results demonstrate that (i) FTIR can be used to track eicosonoid generation in $\mathrm{D}_{8}$-AA stimulated cells; (ii) the intracellular management of $\mathrm{D}_{31}$-PA at highloadings can be elucidated by monitoring lipid biosynthesis through the lipid hydrocarbon peak area signal; (iii) metabolism of lipid pools results in significant protein phosphorylation.
\end{abstract}

(c) 2008 Elsevier B.V. All rights reserved.

\section{Introduction}

In the United Kingdom, prostate cancer (CaP) is the most commonly diagnosed cancer in men and the second most common cause of cancer related death of men [1]. A key factor directly responsible for considerable mobidity and mortality in $\mathrm{CaP}$ patients is metastases to the bone marrow stroma (BMS). Several epidemiological and in vitro moelcular studies have demonstrated that fatty acids (FAs) play a significant role in the progression and development of CaP $[3,4]$. Dennis et al. [2] conducted a detailed retrospective review of 29 epidemiological studies investigating this relationship and identified associations of advanced CaP with intake of total and saturated fat. This is supported by the recent World Cancer Research Fund inquiry, which also reports that a key factor in the development of cancer is total body fat [5]. However, Dennis et al. [2] highlights that currently fat-specific intake in CaP

\footnotetext{
* Corresponding author. Tel.: +44 161446 3232; fax: +44 1614463109

E-mail addresses: EGazi@Picr.man.ac.uk, Ehsan1643@hotmail.com, Egazi@dstl.gov.uk (E. Gazi).
}

progression or prevention remains unclear through patient based studies as a result of inconsistencies in study design. Thus, there is a need for controlled systematic laboratory based studies to facilitate and potentially focus epidemiological investigations.

We have previously used imaging Fourier-Transform infrared (FTIR) microspectroscopy to demonstrate that metastatic CaP cells in bone marrow biopsy tissue with close proximity to adipocytes, give rise to higher lipid hydrocarbon signals relative to those $\mathrm{CaP}$ cells situated further away from these adipocyte-rich locations [6]. Adipocytes are specialised for the synthesis and storage of FAs as triacylglycerides (TAGs) as well as for FA mobilisation through lipolysis [7]. This was followed by in vitro co-culture experiments of PC-3 cells (CaP cell line derived from bone metastases) with human BMS. These experiments also showed that PC-3 cells preferentially located to adipocytes in the presence of numerous haemopoietic cell types and that these tumour cells accumulated numerous cytoplasmic lipid droplets, revealed by Oil-red $\mathrm{O}$ staining for TAG and Nile Red real time fluorescent microscopy [6]. A quantitative measure of this attraction was achieved using Boyden transwell invasion assays. Using this method, we found that invasion of PC-3 cells towards BMS was significantly reduced 
when the experiment was repeated with BMS grown in the absence of hydrocortisone so that adipocyte formation was inhibited [6].

Together, the above findings revealed that PC-3 cells are attracted to adipocytes and that these tumour cells exhibit high lipid signals. From these observations we asked: Could the high levels of lipids measured in metastatic CaP cells in close proximity to adipocytes, be the result of adipocyte to CaP cell lipid translocation or an increased expression of lipogenic enzymes for de novo lipid biosynthesis in the tumour cell? The latter can be induced by growth factors [8] that are also released by adipocytes [9]. To investigate whether lipids stored within adipocytes could be translocated to PC-3 cells, we grew mesenchymal stem cells in media containing deuterated palmitic acid $\left(D_{31}-P A\right)$ during their differentiation into adipocytes. The mature adipocytes, comprising of numerous lipid droplets containing $\mathrm{D}_{31}-\mathrm{PA}$, were then incubated for a further $48 \mathrm{~h}$ in media containing PC-3 cells and no $\mathrm{D}_{31}$-PA. Following this period, the co-culture was fixed and analysed using imaging FTIR microspectroscopy. In the mid-IR spectral window, deuterated FAs can provide a C-D vibrational signal that does not overlap with signals arising from the functional group vibrations of endogenous FAs or other biomolecules. This specific spectral marker for the labelled FA (or its metabolites), was not only observed in high intensity in the adipocytes, confirming $D_{31}-\mathrm{PA}$ uptake and storage in these cells, but was also found to illuminate PC-3 cells following background subtraction. The study demonstrated for the first time, without cell isolation and lipid extraction that tumour cells could uptake lipid stored within adipocytes. Inturn, this highlighted a potential clinical significance for the management of $\mathrm{CaP}$ through diet.

The importance of the role of lipids in the metastatic progression of $\mathrm{CaP}$ was further established by our group through biomolecular profiling of metastatic CaP cells in primary bone marrow tissue sections derived from different patients, with FTIR and histochemistry. This was carried out with comparative biomolecular profiles obtained from high-grade prostate organconfined CaP [10]. This study identified the dominant metabolic processes driving the proliferation of metastatic cells in each of the biopsies studied: Glycerophospholipid synthesis from TAG, glycolipid synthesis as well as glycolysis.

The focus of the present study is to follow on from the above experimental findings by investigating the metabolism of specific isotopically labelled unsaturated and saturated FA's in PC-3 cells in a time-dependent manner using FTIR microspectroscopy. Metabonomic analysis through FTIR microspectroscopy enables a survey of several biomolecules simultaneously (such as lipids, phosphorylated biomolecules and protein secondary structure) to generate hypotheses that may refine targeted molecular studies. The use of deuterated analogues to investigate metabolic activity in tissue has been demonstrated by Wetzel et al. [11]. The authors used deuterated water as an alternative to radioisotopes to study the metabolic activities in various layers of the cerebellum in rat tissue following fixation.

In the present study, arachidonic acid (AA) was chosen as the candidate unsaturated FA since this omega- 6 poly-unsaturated FA ( $\omega-6$ PUFA) has been shown to increase the risk of CaP by promoting the proliferation of malignant prostate epithelial cells. These effects are mediated by the increase in the cyclooxygenase- 2 (COX-2) derived product, prostaglandin $\mathrm{E}_{2}\left(\mathrm{PGE}_{2}\right)[12]$ and the lipoxygenase (LOX) derived products, 5 and 12(S)-HETE (hydroxyeicosatetraenoic acid). Of these eicosanoids, we have previously demonstrated $\mathrm{PGE}_{2}$ to be a potent stimulator of $\mathrm{PC}-3$ invasion [6].

As well as investigating the uptake and metabolic consequences of AA in metastatic CaP, we also study these parameters with a saturated FA, palmitic acid (PA; C16:0). PA is not an essential FA (unlike AA) and is one of the five most abundant FAs comprising adipose tissue or blood serum [13]. Molecular and ecological studies have shown a positive association of saturated fat intake with increased risk and mortality from CaP [13-15]. A molecular based patient study by Mamalakis et al. [16] found a significantly elevated adipose saturated FA composition in CaP patients as opposed to benign prostate hyperplasia ( $\mathrm{BPH})$ patients. Interestingly, the same study also found significantly decreased prostate tissue sum saturated FAs and elevated sum monounsaturated FAs in cancer as opposed to BPH patients. Mamalakis et al. [16] associated these findings to studies at the cellular level demonstrating that a decreased saturation index (SI; the ratio of saturated to monounsaturated FAs) is associated with increased tumour cell fluidity and malignant transformation. Mamalakis et al. [16] also reported that the most pronounced difference between cancer patients and BPH patients was a three-fold elevated level of the desaturated PA metabolite, palmitoleic acid (C16:1), in the former group, highlighting a possible role of this FA in the neoplastic processes.

\section{Experimental}

\subsection{Cell culture}

PC-3 cells were cultured in Ham's F12, 7\% FCS and 1\% LGlutamine on MirrIR slides until 70\% confluent. Following this, one slide of cells were starved in serum-free RPMI media with $1 \%$ LGlutamine for $24 \mathrm{~h}$ and then fixed in 4\% formalin in PBS for $25 \mathrm{~min}$ prior to washing in deionised water for $3 \mathrm{~s}$ to remove the residue PBS [17]. This represented the control slide (no exposure to labelled FA) at $0 \mathrm{~min}$. The remaining PC-3 cells were also starved in serum-free RPMI media with $1 \%$ L-Glutamine for $24 \mathrm{~h}$. These cells were subsequently washed twice in PBS and then exposed to serum-free RPMI with $1 \%$ L-Glutamine, supplemented with $25 \mu \mathrm{M}$ or $100 \mu \mathrm{M} \mathrm{D}_{8}$-AA (Sigma-Aldrich, UK) or $20 \mu \mathrm{M}$ or $50 \mu \mathrm{M} \mathrm{D}_{31}$-PA (CK Gas Products Limited, UK) for 15, 30, 45, 60, 90, 120, 180 and $1440 \mathrm{~min}$. Control slides of PC-3 cells cultured in media with no $D_{31}-P A$ or $D_{8}-A A$ were also prepared for each time-point. The slides were removed from culture media at each of the above timepoints, washed twice with PBS, and fixed using the same protocol as described above.

\subsection{FTIR microspectroscopy}

A sampling aperture size of $600 \mu \mathrm{m} \times 600 \mu \mathrm{m}$ was used to collect IR spectra $(n)$ from 5 locations within each slide. This resulted in FTIR spectra that were representative of the average biochemistry across approximately $\sim 200$ cells at each sampling location, thus $\sim 1000$ cells/sampling time-point. FTIR spectra were recorded in reflectance mode using 256 scans at $4 \mathrm{~cm}^{-1}$ spectral resolution. A background spectrum was collected from a clean MirrIR slide, using 256 scans at $4 \mathrm{~cm}^{-1}$ spectral resolution. FTIR spectra were baseline corrected and normalised to the intensity of the amide I peak.

High definition FTIR microspectroscopic maps were collected at $6.25 \mu \mathrm{m}$ pixel-resolution in rapid-scan mode using a PerkinElmer Spotlight spectrometer with a $16 \times 1 \mathrm{MCT}$ linear array detector. Mid-IR spectra within the range $6000-700 \mathrm{~cm}^{-1}$ were obtained in reflection mode. The background scan was recorded at $8 \mathrm{~cm}^{-1}$ spectral resolution with 75 scans, whereas the sample scan was recorded at $8 \mathrm{~cm}^{-1}$ spectral resolution with 64 scans.

\subsection{Statistics}

Standard errors of the mean $( \pm)$ were calculated for IR measurements collected from different locations on the same 
time-point slide. A general linear model (GLM)-univariate analysis procedure (Two-way analysis of variance) was applied to our $\mathrm{D}_{8}-\mathrm{AA}$ or $\mathrm{D}_{31}-\mathrm{PA}$ uptake data sets to test whether there exists significant difference in $v(C D)$ signals obtained from cells cultured with high or low concentrations of labelled FA, over identical time-points, using the F-statistic. For data sets concerning the temporal fluctuations of lipid, phosphate and protein secondary structure as a result of $\mathrm{D}_{8}-\mathrm{AA}$ or $\mathrm{D}_{31}-\mathrm{PA}$ stimulation at different concentrations, statistical tests were carried out to examine differences in the mean FTIR signals obtained at two different time-points for each condition. For this, a prior check for data normality using the Shapiro-Wilk test was applied to FTIR signal intensities measured for each time-point in the comparison. In the case that data were normally distributed the independent samples $t$-test was applied at $95 \%$ confidence interval. In the case that data showed a skewed distribution, the nonparametric 2 independent samples Mann-Whitney $U$-test was performed at 95\% confidence interval. All statistical tests were carried out using SPSS release 11.0.0.

\section{Results and discussion}

\subsection{Uptake of palmitic acid by PC-3 cells}

Firstly, we studied the influence of different growth medium compositions on FA uptake using $\mathrm{D}_{31}$-PA. Three different culture media were investigated: (i) Ham's F-12 with $7 \%$ fetal bovine serum (FBS) and 1\% L-Glutamine, (ii) RPMI with 7\% FBS and 1\% LGlutamine and (iii) RPMI with 1\% L-Glutamine. Both RPMI and Ham's F-12 are complex mixtures consisting of a range of inorganic salts, amino acids, vitamins, nucleotides and glucose as well as small-molecule precursors. However, it is the Ham's F-12 growth medium with FCS and L-glutamine, which is recommended by the European Collection of Cell Cultures (ECACC) for optimum growth of PC-3 cells [18]. RPMI is recommended by ECACC for culture of CaP cell lines, LNCaP (derived from lymph node metastases) and PNT2-C2 (immortalised normal prostate epithelial cell line) but contains no FAs, unlike Ham's F-12, which contains the $\omega 6$-FA, linoleic acid (LA). Fig. 1A shows FTIR measurements demonstrating the influence of these media on $\mathrm{D}_{31}$-PA uptake by PC-3 cells following $24 \mathrm{~h}$ incubation with $50 \mu \mathrm{M} \mathrm{D}_{31}$-PA. This was carried out by baseline integration of the $v_{a s+s}\left(\mathrm{CD}_{2+3}\right)$ peaks in the spectral region $2250-2050 \mathrm{~cm}^{-1}$ and using the resulting peak areas.

In Fig. $1 \mathrm{~A}$ we find that FBS supplementation into the media resulted in a significant reduction in $\mathrm{D}_{31}$-PA uptake compared with cells incubated in media without FBS $(p<0.05)$. The main constituent of FBS is the globular protein, albumin, which may influence $\mathrm{D}_{31}$-PA uptake by reducing albumin bound $\mathrm{D}_{31}-\mathrm{PA}$ interactions with the plasma membrane, in-turn preventing passive diffusion of $\mathrm{D}_{31}$-PA through the plasma membrane and/ or interactions of $D_{31}-\mathrm{PA}$ with membrane-bound FA transporter proteins [19]. FBS is also rich in an array of other proteins, FAs and growth stimulatory factors [20] that may support growth and circumvent the requirement for $\mathrm{D}_{31}$-PA uptake. Therefore, to study the uptake and metabolism of $\mathrm{D}_{31}$-PA by PC-3 cells, we used the simplified medium composition of RPMI in the absence of FBS, therefore FA free media, enabling accurate measurement of $D_{31}-\mathrm{PA}$ uptake and metabolism by PC-3 cells without the background contamination of undefined amounts of FA from the media.

The subcellular preservation and localisation of $\mathrm{D}_{31}$-PA into PC3 cells was assessed using FTIR microspectroscopic imaging. Fig. 1B shows an optical photomicrograph of PC-3 cells on MirrIR substrate, following exposure to $50 \mu \mathrm{M} \mathrm{D}_{31}$-PA for $24 \mathrm{~h}$. Fig. $1 \mathrm{C}$ shows the distribution of the integrated intensity of the phosphate diester $\left(v_{a s}\left(\mathrm{PO}_{2}\right) ;\left(1274-1181 \mathrm{~cm}^{-1}\right)\right)$ peak area. For cells 1 and 2 in the optical image, it is clearly observed that the most intense phosphate signals localise at the nuclei due to the high concentration of nucleic acids present at this location. The integrated lipid hydrocarbon peak area $\left(v_{a s+s}\left(\mathrm{CH}_{2+3}\right)\right.$; 3004$2837 \mathrm{~cm}^{-1}$ ) signal localises with greater intensity at the cytoplasmic locations due to the presence of cellular organelles. Accepting that the cell thickness does not change significantly across the cell, we observe that the most intense $v_{a s+s}\left(\mathrm{CD}_{2+3}\right)$ signal co-localises with the lipid hydrocarbon signal at the cytoplasmic locations, suggesting the subcellular localisation of $D_{31}$-PA or its metabolites is predominately in the cytoplasm. To demonstrate spectral quality, Fig. 1F shows raw FTIR spectra taken from the nucleus (Location 1) and cytoplasm (Location 2) of PC-3 cell 2 (See Fig. 1E).

Fig. 2 shows a graph of intracellular $v_{s+a s}\left(\mathrm{CD}_{2+3}\right)$ signal intensities as a function of time in PC-3 cells incubated with $20 \mu \mathrm{M}$ or $50 \mu \mathrm{M} D_{31}$-PA. A pairwise comparison of $v_{s+a s}\left(\mathrm{CD}_{2+3}\right)$ signal intensities at each time-point for cells incubated with 20 and $50 \mu \mathrm{M} \mathrm{D} \mathrm{D}_{31}$-PA reveals a significant difference only at $24 \mathrm{~h}$ (1440 min) with $p=0.00$. It is possible that this difference at 1440 min may be the result of total/near complete $D_{31}$-PA uptake by the PC-3 cells from the media that was initially supplemented with $20 \mu \mathrm{M} \mathrm{D}_{31}$-PA. In contrast, at the 1440 min time-point, the $50 \mu \mathrm{M} \mathrm{D}_{31}$-PA supplemented media may still contain available $D_{31}-P A$ for cell consumption and this may reflect the high intracellular $v_{s+a s}\left(\mathrm{CD}_{2+3}\right)$ signal intensity measured from these cells.

\subsection{The temporal effects of $D_{31}-P A$ exposure to endogenous biomolecules in PC-3 cells}

The FTIR technique enables us to probe fluctuations in several biomolecules simultaneously. Thus, in addition to monitoring $\mathrm{D}_{31^{-}}$ PA uptake directly using the $v_{s+a s}\left(\mathrm{CD}_{2+3}\right)$ signal, it is also possible to temporally determine the effects of this exposure on phosphate activity (integrated peak area $1274-1181 \mathrm{~cm}^{-1}$ ), protein secondary structures (amide I frequency) and endogenous-non-isotopically labelled lipid biosynthesis (integrated peak area 3004$2837 \mathrm{~cm}^{-1}$ ). The temporal fluctuations in these biomolecular domains were studied in PC-3 cells following incubation with 20 and $50 \mu \mathrm{m} \mathrm{D} \mathrm{D}_{31}-\mathrm{PA}$ in serum-free culture media (RPMI) and compared with the control (PC-3 cells incubated in identical conditions but without $\mathrm{D}_{31}-\mathrm{PA}$ ) (Fig. 3A-C). Fig. 3D shows a typical raw FTIR spectrum taken from the dataset to illustrate baseline correction and integration to obtain peak area values for the lipid and phosphate signals.

Firstly, statistical tests (two-way ANOVA followed by Tukey multiple comparisons test) were used to identify whether each of the IR signals (protein amide I frequency shifts, endogenous lipid intensities, phosphate intensities) obtained over the $24 \mathrm{~h}$ period for control cells were significantly different from those obtained from $D_{31}$-PA stimulated cells. These tests were also carried out to determine whether the measured signals were different between cells stimulated with high and low concentrations of $\mathrm{D}_{31}$-PA. Using this statistical test we found that combinations of control $v s$. $20 \mu \mathrm{M} \mathrm{D}_{31}$-PA stimulated cells, control vs. $50 \mu \mathrm{M} \mathrm{D}_{31}-\mathrm{PA}$ stimulated cells and $20 \mu \mathrm{M} \mathrm{D} \mathrm{D}_{31}$-PA stimulated cells vs. $50 \mu \mathrm{M}$ $D_{31}$-PA stimulated cells gave rise to protein shifts and phosphate signals that were significantly different with $p \leq 0.05$. For the lipid signal, we found that all of the combinations stated above were also significantly different $(p \leq 0.05)$ except for the control $v s$. $20 \mu \mathrm{M} \mathrm{D}{ }_{31}$-PA stimulated cells $(p=0.252$ ), this will be discussed further below. These results demonstrate that addition of $D_{31}-\mathrm{PA}$ has a significant effect on the biochemistry of the cells over the duration of the time-course experiment compared with the control. 
A.

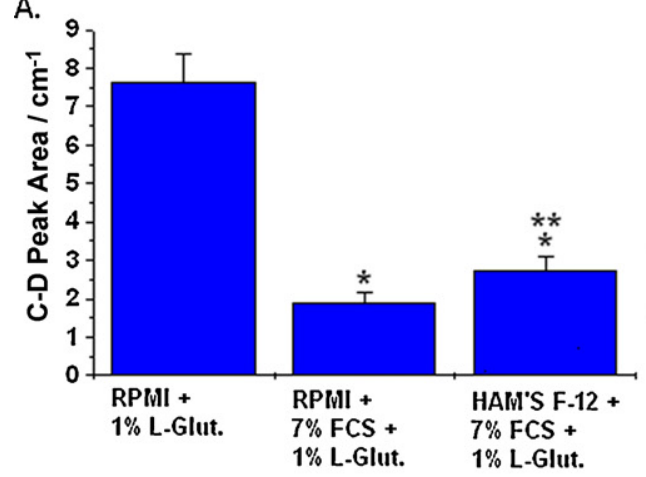

Growth Medium
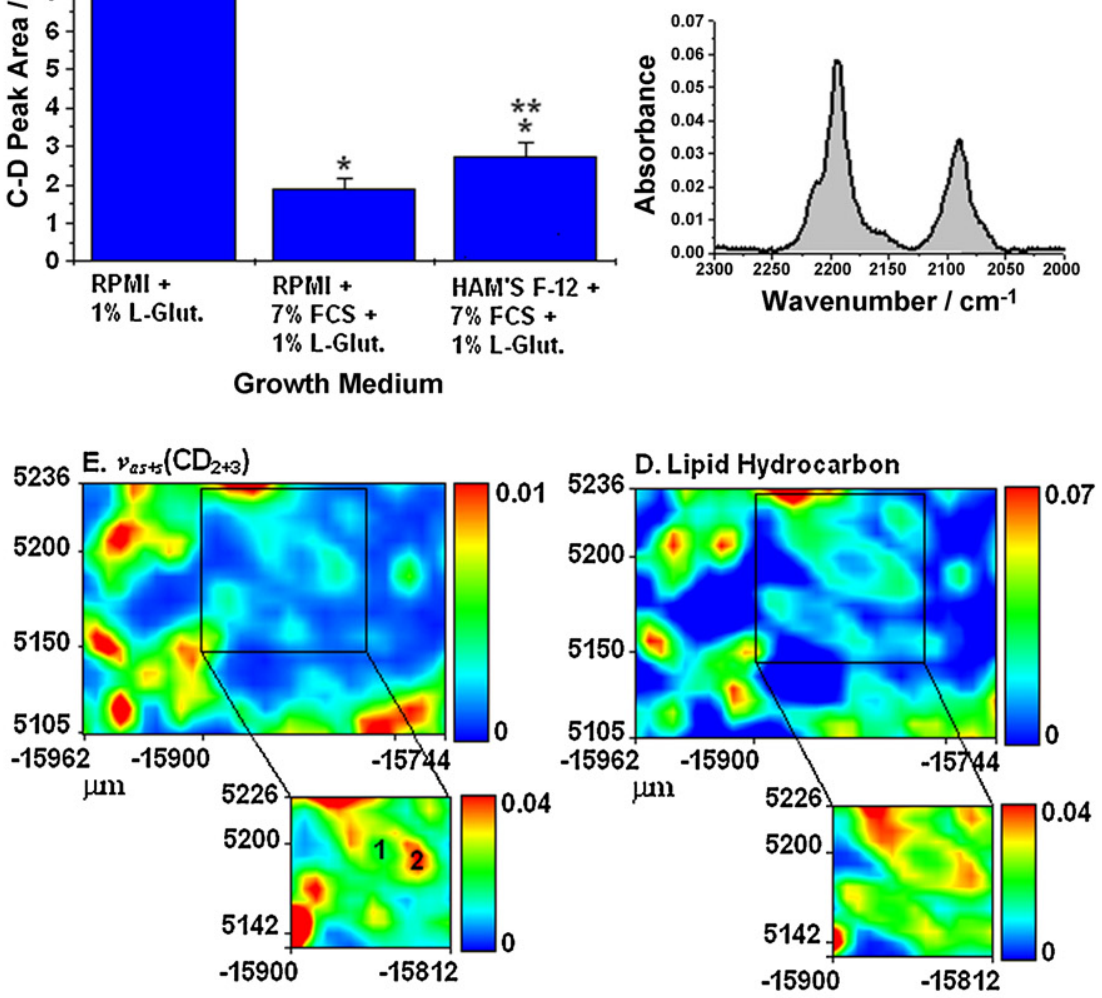

B. Optical

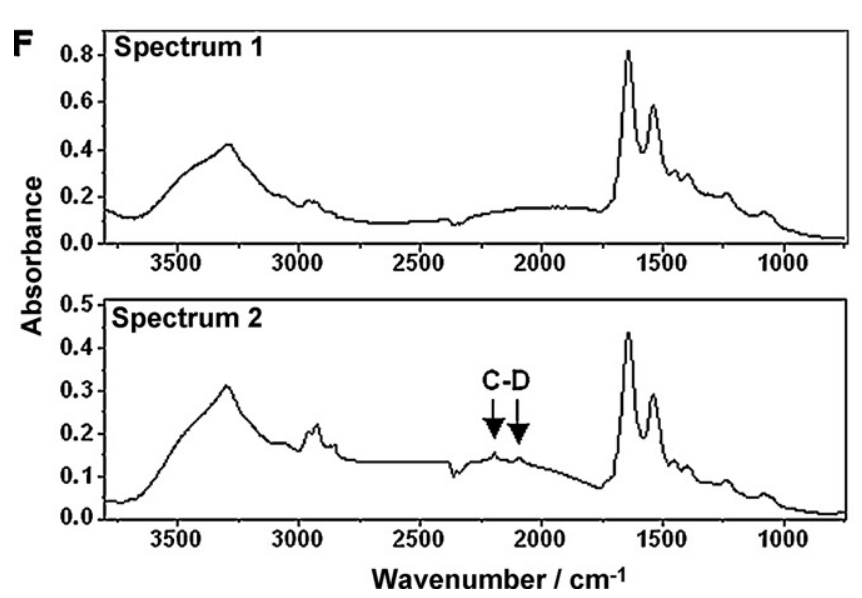

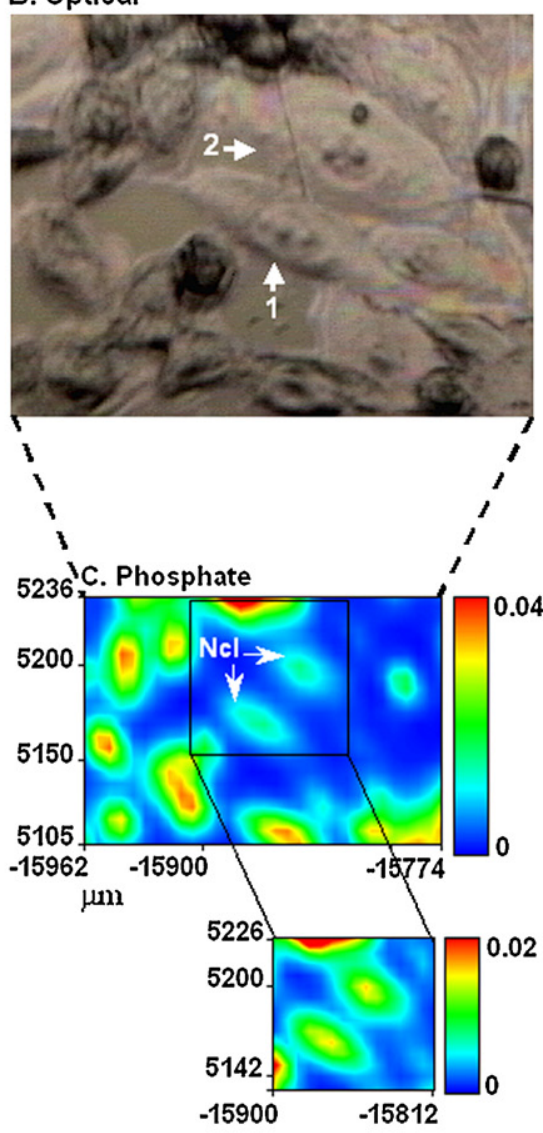

$-15900$

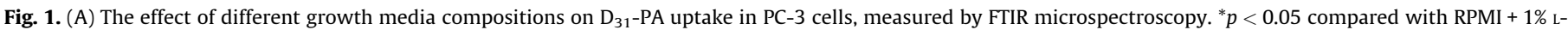

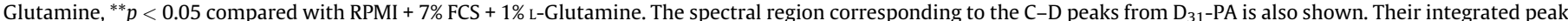

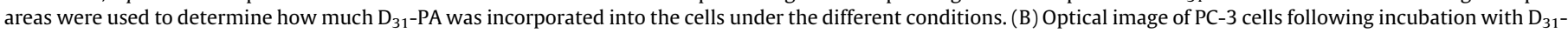

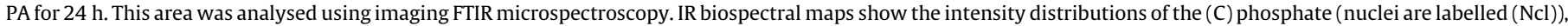

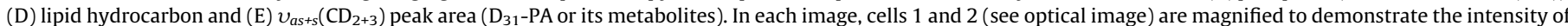

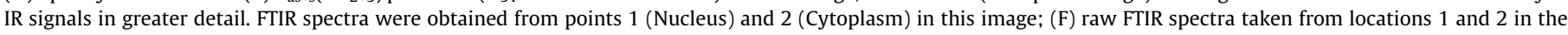
$v_{a s+s}(\mathrm{CD})_{2+3}$ intensity image.

Following this assessment, fluctuations in the different biomolecular domains between time-points for each culture condition were studied. We found that the endogenous lipid signal in the control PC-3 cells initially fell and is induced by metabolic/cytokine/growth factor imbalance resulting from the exchange of media to serum-free RPMI media at the zero minute time-point (Fig. 3A). Conversely, cells incubated with $\mathrm{D}_{31}-\mathrm{PA}$ exhibited a rapid rise in endogenous lipids within $15 \mathrm{~min}$ of exposure. Since the incubation media (RPMI) contains no FAs, this increase in lipid content must be due to de novo biosynthesis. This correlates with previous reports demonstrating that the majority of FAs in tumour cells are derived from de novo FA synthesis and in contrast to normal tissue, human tumours display a higher rate of de novo FA synthesis [8]. Also, it has been shown that lipotoxicity from accumulation of long chain FAs is specific for saturated FAs such as PA [21]. This selectivity arises from the generation of molecules such as reactive oxidative species or the de novo synthesis of ceramide, among others [21]. Listenberger et al. [21] 


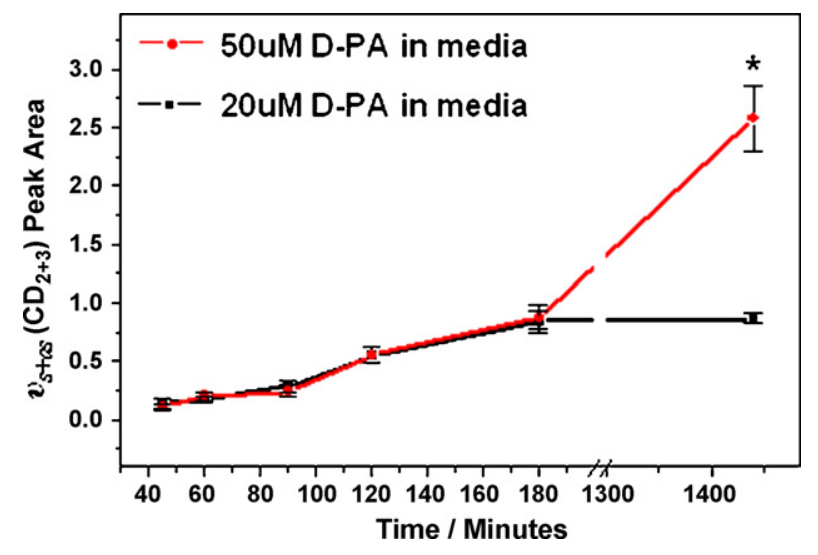

Fig. 2. A graph to show mean intracellular $v_{s+a s}\left(\mathrm{CD}_{2+3}\right)$ signal intensities $( \pm \mathrm{SE})$ as a function of time in PC-3 cells incubated with $20 \mu \mathrm{M}$ or $50 \mu \mathrm{M} \mathrm{D}_{31}$-PA. Standard error of the mean bars are displayed. Test for significance between $v_{s+a s}\left(\mathrm{CD}_{2+3}\right)$ signals were obtained for 20 and $50 \mu \mathrm{M} \mathrm{D} \mathrm{D}_{31}$-PA stimulated cells at each time-point, where ${ }^{*}$ is significant when $p \leq 0.05$.

have shown that in Chinese hampster ovary (CHO) cells, endogenously generated unsaturated FAs rescue PA-induced apoptosis by promoting PA incorporation into TAG. It may be the case that this mechanism is initiated in the PC-3 cells shortly following exposure to $\mathrm{D}_{31}$-PA and is responsible for the significant up-regulation in endogenous lipids. To the author's knowledge, this mechanism has not previously been proposed or demonstrated for CaP cells.

For PC-3 cells incubated with $50 \mu \mathrm{m} \mathrm{D}_{31}-\mathrm{PA}$, the initial rise in endogenous lipid signal was followed by a fall, which we attribute to metabolic breakdown into adenosine triphosphate (ATP), which is a major product of lipid metabolism. This notion is supported by a phosphate spike at $30 \mathrm{~min}$ (Fig. 3B) accompanied by a marked shift in the amide I frequency (Fig. 3C), together indicating protein phosphorylation. Although protein phosphorylation is also observed in the control cells at a later time-point (45 min, Fig. 3C), it is not known at this juncture whether the proteins that are phosphorylated are the same as those phosphorylated when PC-3 cells are incubated with $\mathrm{D}_{31}$-PA. This requires further metabolomic and phosphopeptide-based investigations.

Earlier in this section we reported that the overall trend in endogenous lipid signal between control vs. $20 \mu \mathrm{M} \mathrm{D}_{31}-\mathrm{PA}$ stimulated cells were not significantly different. However, we can identify significant differences in endogenous lipid signals between these conditions at earlier time-points (30 and $45 \mathrm{~min}$ ). Together, this data indicates an expected smaller perturbation to the metabolites in cells exposed to the lower concentration of $\mathrm{D}_{31^{-}}$ PA compared with exposure to higher concentrations. The endogenous lipid intensity in cells exposed to $20 \mu \mathrm{M} \mathrm{D}_{31}$-PA demonstrates a maximum at 45 min post $\mathrm{D}_{31}$-PA induction (Fig. 3A). This time-delay in reaching a lipid maximum, compared with cells incubated with $50 \mu \mathrm{M} \mathrm{D}_{31}$-PA, could suggest that if the uptake of $\mathrm{D}_{31}$-PA into the cell is greater with higher gradients, then the critical-point at which lipid biosynthesis is initiated to sequester excess $D_{31}$-PA must also be delayed for culture media containing lower concentrations of $\mathrm{D}_{31}$-PA. Interestingly, the $50 \mu \mathrm{M} \mathrm{D}_{31}$-PA exposed cells show a significantly higher maximum lipid signal compared with the $20 \mu \mathrm{M} \mathrm{D}_{31}$-PA exposed cells, within this period $(0-45 \mathrm{~min})$. This suggests that endogenous lipid demand is greater in cells incubated with higher concentrations of $D_{31}-\mathrm{PA}$ compared with cells incubated in lower concentrations of $\mathrm{D}_{31}$-PA. This supports the assumption that a greater influx of $\mathrm{D}_{31}$-PA requires for a greater number of synthesised lipids. Unfortunately, it was not possible to measure the $v_{s+a s}\left(\mathrm{CD}_{2+3}\right)$

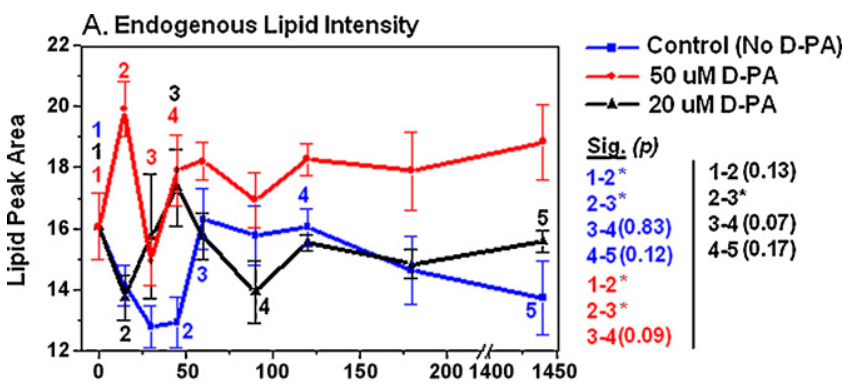

B. Phosphate Intensity
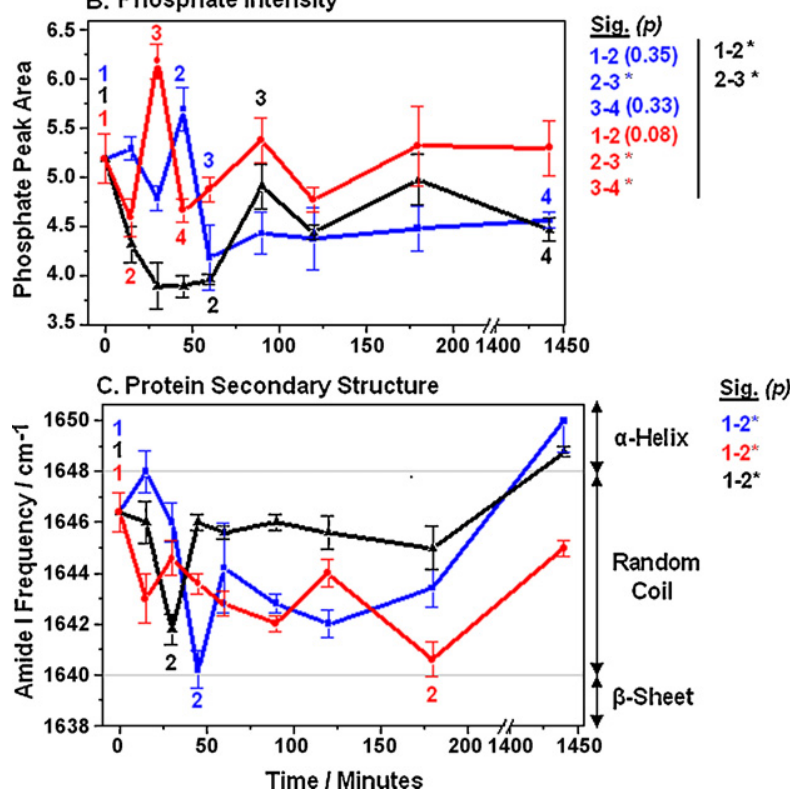

Fig. 3. Temporal fluctuations in various biomolecular domains probed by FTIR, for PC-3 cells exposed to $20 \mu \mathrm{M}$ or $50 \mu \mathrm{M} \mathrm{D}_{31}$-PA or no $\mathrm{D}_{31}$-PA (Control). (A) Endogenous mean lipid hydrocarbon peak area intensities $( \pm \mathrm{SE})$; (B) mean phosphate diester peak area $\left(v_{a s}\left(\mathrm{PO}_{2}\right)\right)$ intensities $( \pm \mathrm{SE}) ;(\mathrm{C})$ amide I frequency shifts $( \pm \mathrm{SE}) .{ }^{*}$ Significance test values between time-points are displayed, where ${ }^{*}$ is significant when $p \leq 0.05$; (D) typical raw FTIR spectrum taken from the dataset to illustrate baseline correction and integration to obtain peak area values for the lipid and phosphate signals.

intensity at time-points below 45 min since the amount in the cells was below the detection limit with this experimental set-up.

The anticorrelation of lipid with phosphate intensity was not only observed in cells incubated with $50 \mu \mathrm{M} \mathrm{D}_{31}-\mathrm{PA}$, but also for cells incubated with $20 \mu \mathrm{M} \mathrm{D} \mathrm{D}_{31}$-PA between 45 and $90 \mathrm{~min}$ (Fig. 3B). However, unlike the control and cells exposed to $50 \mu \mathrm{M}$ $\mathrm{D}_{31}-\mathrm{PA}$, the rise in phosphate signal did not induce a concomitant change in bulk protein secondary structure. In contrast, we observed a more gradual change in protein secondary structure over-time for cells incubated with $20 \mu \mathrm{M} \mathrm{D}_{31}$-PA. It may be the case that these phosphates are partitioned between other cellular processes such as phospholipid generation as well as protein phosphorylation, resulting in a more subtle change to the latter.

FTIR microspectroscopy can directly probe structural modifications of $\mathrm{D}_{31}$-PA resulting from its metabolism by the PC-3 cells. This is carried out using the $v_{a s}\left(\mathrm{CD}_{2}\right): v_{a s}\left(\mathrm{CD}_{3}\right)$ signal intensity, which can determine changes in $\mathrm{D}_{31}$-PA chain length (higher $v_{a s}\left(\mathrm{CD}_{2}\right): v_{a s}\left(\mathrm{CD}_{3}\right)$ values indicate longer $\mathrm{FA}$ chains). The inset in Fig. 4 shows the method by which the $v_{a s}\left(\mathrm{CD}_{2}\right)$ and $v_{a s}\left(\mathrm{CD}_{3}\right)$ values are obtained, which is based on peak height. Note that for PC-3 cells incubated with $20 \mu \mathrm{M} \mathrm{D}_{31}$-PA, we found that the subtle $v_{a s}\left(\mathrm{CD}_{3}\right)$ signal that exists as a shoulder on the $v_{a s}\left(\mathrm{CD}_{2}\right)$ peak was not resolvable in spectra obtained at the 90 min and 120 min sampling 


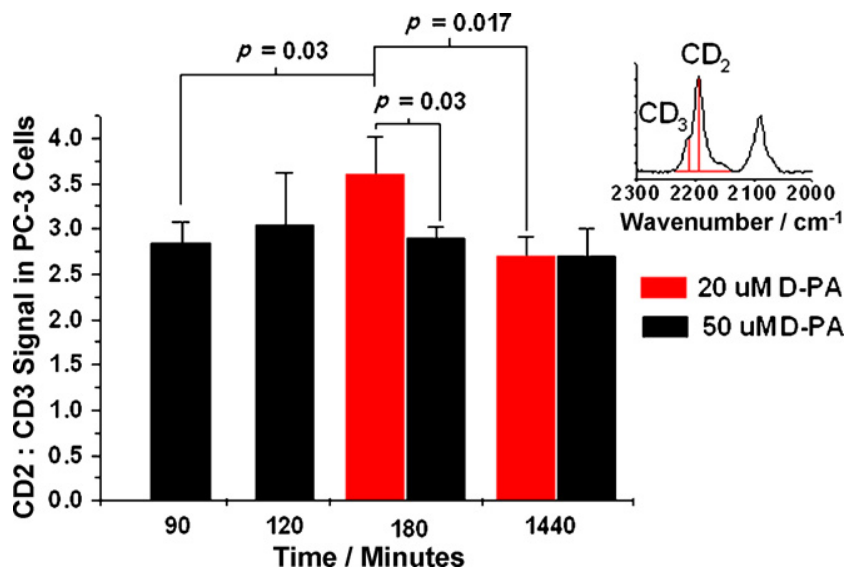

Fig. 4. $\mathrm{D}_{31}-\mathrm{PA}$ metabolism monitored by temporal changes in $v_{a s}\left(\mathrm{CD}_{2}\right): v_{a s}\left(\mathrm{CD}_{3}\right)$ values $\left( \pm \mathrm{SE}\right.$ ) for cells incubated with 50 and $20 \mu \mathrm{M} \mathrm{D} \mathrm{D}_{31}$-PA. Inset shows the $v(\mathrm{CD})_{2 / 3}$ region with baseline and heights used to obtain the ratio values.

time-points. Fig. 4 shows that PC-3 cells incubated with $20 \mu \mathrm{M}$ $\mathrm{D}_{31}$-PA for $3 \mathrm{~h}$ exhibit significantly higher $v_{a s}\left(\mathrm{CD}_{2}\right): v_{a s}\left(\mathrm{CD}_{3}\right)$ values compared with cells exposed to $50 \mu \mathrm{M} \mathrm{D}_{31}$-PA for 90 and $180 \mathrm{~min}$ ( $p=0.03$ and $p=0.02$, respectively). This data indicates lower metabolism of $\mathrm{D}_{31}$-PA in PC-3 cells exposed to $20 \mu \mathrm{M} \mathrm{D}_{31}-\mathrm{PA}$ compared with cells incubated with $50 \mu \mathrm{M} \mathrm{D}_{31}$-PA. This low rate of $\mathrm{D}_{31}$-PA metabolism may be due to the requirement for lipid derived products being satisfied by metabolism of endogenous lipids, which we find to provide lower values ( $\geq 60 \mathrm{~min}$ ) in the cells exposed to $20 \mu \mathrm{M} \mathrm{D}_{31}$-PA compared with cells exposed to $50 \mu \mathrm{M}$ $\mathrm{D}_{31}$-PA (Fig. $3 \mathrm{~A}$ ). At 1440 min post $\mathrm{D}_{31}-\mathrm{PA}$ induction, we find that there is a significant reduction in the $v_{a s}\left(C_{2}\right): v_{a s}\left(C_{3}\right)$ values for cells stimulated with $20 \mu \mathrm{M} \mathrm{D}_{31}-\mathrm{PA}$, generating $v_{a s}\left(\mathrm{CD}_{2}\right): v_{a s}\left(\mathrm{CD}_{3}\right)$ values similar to those stimulated with $50 \mu \mathrm{M} \mathrm{D}_{31}-\mathrm{PA}$ for 1440 min (Fig. 4). Thus, $\mathrm{D}_{31}$-PA initially stored upon exposure may be metabolised eliciting the reduction in $v_{a s}\left(\mathrm{CD}_{2}\right): v_{a s}\left(\mathrm{CD}_{3}\right)$ values between 90 and 1440 min time-points for the $20 \mu \mathrm{M} \mathrm{D}_{31^{-}}$ PA stimulated cells. This reduction supports the inference made above, in which we hypothesised significant depletion of $D_{31}-P A$ in the culture media between 90 and $1440 \mathrm{~min}$. Thus, between these time-points metabolised $\mathrm{D}_{31}$-PA may not have been replenished in the cells.

Note that in the above experiments, the reproducibility of FTIR signals obtained from cells within each time-point has been measured by the standard error. Further investigations are required to address the reproducibility of temporal fluctuations in FTIR signals from cells exposed to $D_{31}$-PA in independent experiments.

\subsection{Uptake and metabolism of arachidonic acid by PC-3 cells}

Previously, we determined the rate of AA uptake into PC-3 cells since it is known to decrease in the adipose tissue of CaP patients [6]. In that experiment, PC-3 cells were cultured in the presence of $10 \mu \mathrm{M}$ AA and stained with Nile Red for fluorescence-assisted cell sorting (FACS) analysis. Nile Red is a fluorescent tracer that stains all neutral lipids such as TAG, cholesteryl esters (lipoproteins) and phospholipids [22]. Using this method, we observed rapid uptake of AA from the local environment, reaching a maximum level at $45 \mathrm{~min}$. Following this, we observed decay in the fluorescence signal that suggested metabolism of AA into $\mathrm{PGE}_{2}$ or HETEs.

To compliment the Nile Red experiments, we studied uptake and metabolism of AA using FTIR and deuterated AA ( $\left.D_{8}-A A\right)$ (Fig. 5). This was carried out because Nile Red is not specific to AA and excludes the measurement of free FAs. The latter is important for including into measurements of AA intracellular concentrations, since unesterified AA is the structural form of this FA upon initial uptake into the PC-3 cell or following release from plasma membrane stores by phospholipases $A_{2}$ [23]. $D_{8}$-AA uptake and or that of its metabolites were followed using the ( $=C-D)$ intensity by integration of the $v(=\mathrm{C}-\mathrm{D})$ peak at $\sim 2250 \mathrm{~cm}^{-1}$ (Fig. 5A). This signal appears $\sim 54 \mathrm{~cm}^{-1}$ higher than the $v(C D)$ peaks of $D_{31}-P A$. Since the deuterium atoms in $\mathrm{D}_{31}-\mathrm{PA}$ are located along the carbon chain, they can give rise to the $v_{s}\left(\mathrm{CD}_{2}\right)$ and $v_{a s}\left(\mathrm{CD}_{2}\right)$ vibrations, as well as to the $v_{s}\left(C D_{3}\right)$ and $v_{a s}\left(C_{3}\right)$ vibrations from the terminal carbon. In contrast, the deuterium atoms in $D_{8}-A A$ are located only at the double bonds (Fig. 5A). The unique vibrational signatures for these lipids, highlights the potential pharmokinetic use of FTIR to simultaneously study uptake and metabolism of both saturated and unsaturated FAs in tumour cells.

Fig. 5B shows the mean intracellular $v(=\mathrm{C}-\mathrm{D})$ signal intensities as a function of time in PC-3 cells incubated with $25 \mu \mathrm{M}$ or $100 \mu \mathrm{M}$

A.<smiles>[2H]C(=O)CCCCCCCCCC(=O)O</smiles><smiles>[2H]C([2H])([2H])C([2H])([2H])C([2H])([2H])C([2H])([2H])C([2H])([2H])C([2H])([2H])C([2H])([2H])C([2H])([2H])C([2H])([2H])C([2H])([2H])C([2H])([2H])C([2H])([2H])C(=O)O</smiles>

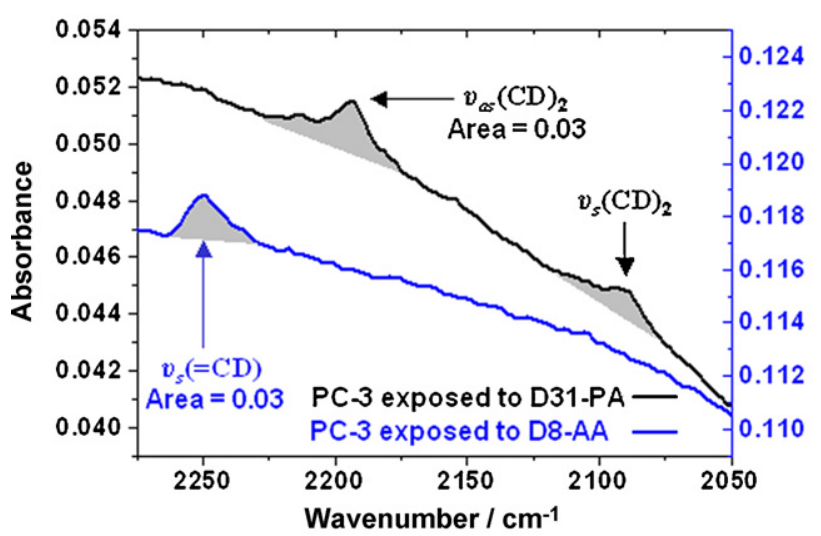

B. FTIR - D-AA Uptake / Metabolism

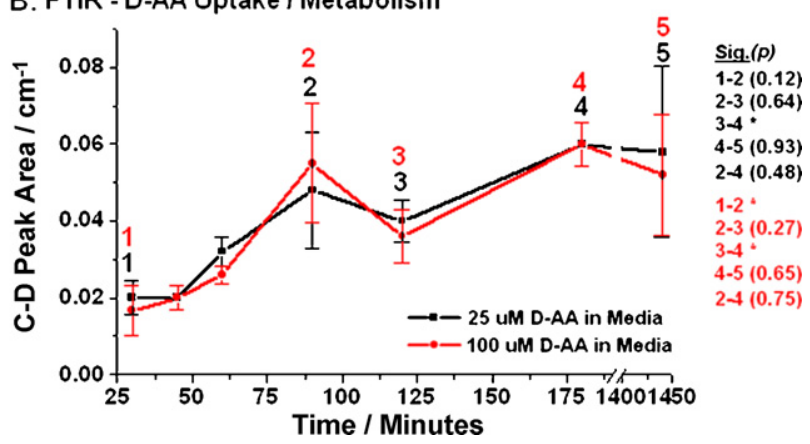

Fig. 5. (A) Molecular structures of $D_{8}-A A$ or $D_{31}-P A$. Raw FTIR spectra in the $C-D$ region of $P C-3$ cells exposed to $\mathrm{D}_{8}-\mathrm{AA}$ or $\mathrm{D}_{31}-\mathrm{PA}$ : The difference in the number of peaks observed in each spectrum for this spectral region arises from the differences in deuterated sites in the molecular structure of $D_{8}-A A$ and $D_{31}-P A ;(B)$ a graph to show mean intracellular $v(=\mathrm{C}-\mathrm{D})$ signal intensities $( \pm \mathrm{SE})$ as a function of time in PC3 cells incubated with $25 \mu \mathrm{M}$ or $100 \mu \mathrm{M} \mathrm{D}_{8}$-AA. Significance test values between timepoints are displayed, where ${ }^{*}$ is significant when $p \leq 0.05$. 
$\mathrm{D}_{8}-\mathrm{AA}$. We found that the $v(=\mathrm{C}-\mathrm{D})$ signals could be measured at time-points $\geq 30 \mathrm{~min}$. Prior to $30 \mathrm{~min}$, signal intensity of the $v(=\mathrm{C}-$ D) peak could not be detected. We observe a significant rise in $v(=\mathrm{C}-\mathrm{D})$ signal between 30 and $90 \mathrm{~min}$ for $100 \mu \mathrm{M} \mathrm{D}_{8}$-AA exposed cells. This rise is also demonstrated by the mean $v(=\mathrm{C}-\mathrm{D})$ signal for cells incubated with $25 \mu \mathrm{M} \mathrm{D} \mathrm{D}_{8}-\mathrm{AA}$, although not significant at $p=0.05$. Following this, there is a fall in the mean $v(=\mathrm{C}-\mathrm{D})$ signal between 90 and 120 min for both 25 and $100 \mu M D_{8}$-AA exposed cells. Although this fall is not significant, there does exist a significant increase in $v(=\mathrm{C}-\mathrm{D})$ signal between 120 and $180 \mathrm{~min}$ for both 25 and $100 \mu \mathrm{M} \mathrm{D}_{8}$-AA exposed cells. There is no significant difference in the $v(=\mathrm{C}-\mathrm{D})$ signal between 90 and $180 \mathrm{~min}$ or $180 \mathrm{~min}$ and $24 \mathrm{~h}$ for cells exposed to 25 and $100 \mu \mathrm{M} \mathrm{D}_{8}-\mathrm{AA}$.

The time-point of maximum AA uptake prior to metabolism was determined as $45 \mathrm{~min}$ by the Nile Red method [4]. However, the FTIR data demonstrates a maximum at 90 min (Fig. 5B). This difference is due to the different molecules being measured by FTIR compared with the Nile Red technique, since FTIR records signals from stored intracellular AA as well as its metabolic products that may be synthesised following the 45 min time-point, accumulatively contributing to a net increase in $v(=\mathrm{C}-\mathrm{D})$ signal at $90 \mathrm{~min}$. The AA-derived metabolite fraction cannot be detected using the Nile Red measurement. The fall in $v(=\mathrm{C}-\mathrm{D})$ signal post $90 \mathrm{~min} \mathrm{D}_{8^{-}}$ AA stimulation, can be due to the efflux of $D_{8}-A A$-derived eicosanoids such as $\mathrm{PGE}_{2}$, from the PC-3 cells into the extracellular environment, to convey their biological effects in an autocrine or paracrine manner. Supporting evidence for this is given by HughesFulford [24] and Chen et al. [25], who observed a significant increase in COX-2 protein activity and $\mathrm{PGE}_{2}$ synthesis within three hours of exposure to AA. In comparison with lipid uptake data obtained from PC-3 cells incubated with $\mathrm{D}_{31}$-PA (Fig. 2), we do not find any reduction in $v_{s+a s}\left(\mathrm{CD}_{2+3}\right)$ signal over the time-points sampled for these cells. In-turn, this indicates the absence of $D_{31^{-}}$ PA derived metabolites secreted from the cell.

At $180 \mathrm{~min}$, we observe a second rise in $v(=\mathrm{C}-\mathrm{D})$ signal, reaching a level that is not significantly different from that measured at the $90 \mathrm{~min}$ time-point (Fig. 5B). This indicates regulated uptake of $D_{8}-A A$. Supporting this notion is the comparison of $v(=\mathrm{C}-\mathrm{D})$ signal intensities for PC-3 cells exposed to 25 and $100 \mu \mathrm{M} \mathrm{D}_{8}$-AA over all time-points. Two-way analysis of variance was applied to the data set in Fig. $5 \mathrm{~B}$ to test whether there exists a significant difference in $v(=\mathrm{C}-\mathrm{D})$ signals obtained from cells cultured in 25 and $100 \mu \mathrm{M} \mathrm{D}_{8}-\mathrm{AA}$, over identical time-points. This confirmed that combinations of culture media (with $25 \mu \mathrm{M}$ or $100 \mu \mathrm{M} \mathrm{D}_{8}-\mathrm{AA}$ ) and time gave rise to no significant difference $(F=0.097, p=0.996)$ in $v(=C-D)$ signals. Thus, the amount of $\mathrm{D}_{8^{-}}$ AA in PC-3 cells is tightly regulated, even in the presence of high concentrations of $\mathrm{D}_{8}-\mathrm{AA}$ in the extracellular environment. Stringent control of intracellular unesterified AA has previously been reported for other mammalian cells [23].

\section{Conclusions}

In this paper we have demonstrated that FTIR microspectroscopy can be used to measure the relative uptake of a labelled FA in tumour cells under different environmental conditions, without cell isolation or lipid extraction and enrichment. We observed that incorporation of $\mathrm{D}_{31}$-PA into the $\mathrm{PC}-3$ cell is not as tightly regulated as was found for cells incubated with $D_{8}-A A$, since the level of $D_{31^{-}}$ PA in PC-3 cells rose as a function of availability in the culture medium. This study also confirms our previous findings [6] and others [12] that AA is rapidly taken up from the extracellular environment and metabolised within three hours. This can be correlated with the low levels of AA found in prostate cancer patients.
FTIR microspectroscopy is well-suited for surveying several biomolecular domains simultaneously for generating hypotheses to refine targeted molecular studies. This was demonstrated by temporal FTIR analysis of $\mathrm{D}_{8}$-AA uptake and metabolism, where we can identify the time-point corresponding to significant eicosanoid generation, as well as differences in the response of PC-3 cells to low and high concentrations of $\mathrm{D}_{8}-\mathrm{AA}$ or $\mathrm{D}_{31}-\mathrm{PA}$. We found that stimulation of PC-3 cells with the saturated FA, $D_{31}-\mathrm{PA}$, resulted in significant de novo lipid biosynthesis, which may be associated with $\mathrm{D}_{31}$-PA channelling into TAG pools. The time taken to develop these lipid pools is dependent on the dose of $\mathrm{D}_{31}$-PA administered to the PC-3 cells. $\mathrm{D}_{31}$-PA stimulation also results in significant protein phosphorylation within $3 \mathrm{~h}$ of exposure to PC-3 cells, which may be associated with the metabolic breakdown of these biosynthesised lipids. Thus, if the formation of TAG pools is required for PC-3 cell survival to high intracellular concentrations of $\mathrm{D}_{31}-\mathrm{PA}$ or $\mathrm{D}_{8}-\mathrm{AA}$, as is the case for $\mathrm{CHO}$ cells [21], then lipogenic enzymes involved in the synthesis of TAG may be targets for therapeutic intervention.

\section{Acknowledgements}

We gratefully thank the Association of International Cancer Research (AICR) for funding (E.G) and Dr. James Nicholson (Daresbury Laboratory, UK) for use of the Spotlight instrument.

\section{References}

[1] Cancer Research UK. http://info.cancerresearchuk.org/cancerandresearch/cancers/prostate/ Accessed 6th January 2008. Page Updated March 2007.

[2] L.K. Dennis, L.G. Snetselaar, B.J. Smith, R.E. Stewart, M.E.C. Robbins, Am. J. Epidemiol. 160 (2004) 436.

[3] E. Giovanuccie, E.B. Rimm, C.G. Chute, I. Kawachi, G.A. Colditz, M.J. Stampfer, W.C. Willett, Am. J. Epidemiol. 138 (1993) 620.

[4] A.E. Norrish, C.M. Skeaff, G.L.B. Arribas, S.J. Sharpe, R.T. Jackson, Brit. J. Cancer 81 (1999) 1238.

[5] World Cancer Research Fund/American Institute for Cancer Research, Food, Nutrition, Physical Activity, and the Prevention of Cancer: a Global Perspective, AICR, Washington, DC, 2007.

[6] M.D. Brown, C.A. Hart, E. Gazi, S. Bagley, N.W. Clarke, Brit. J. Cancer 94 (2006) 842.

[7] J.K. Sethi, G.S. Hotamisligril, Cell Dev. Biol. 10 (1999) 19.

[8] J.V. Swinnen, K. Brusselmans, G. Verhoeven, Curr. Opin. Clin. Nutr. Metab. Care 6 (2006) 358.

[9] G. Frühbeck, J. Gomez-Ambrosi, F.J. Muruzabal, M.A. Burrell, Am. J. Physiol. Endocrinol. Metab. 280 (2001) E827.

[10] E. Gazi, J. Dwyer, N.P. Lockyer, P. Gardner, J.H. Shanks, J.-A. Roulson, C.A. Hart, N.W. Clarke, M.D. Brown, Anal. Bioanal. Chem. 387 (2007) 1621.

[11] D. Wetzel, D. Slatkin, S. Levine, Cell Mol. Biol. 44 (1998) 15.

[12] M. Hughes-Fulford, R.R. Tjandrawinata, C.F. Li, S. Sayyah, Carcinogenesis 26 (2005) (2005) 1520

[13] S. Harvei, K.S. Bjerve, S. Tretli, E. Jellum, T.E. Robsahm, L. Vatten, Int. J. Cancer 71 (1997) 545.

[14] S. Franceschi, Epidemiology 5 (1994) 271.

[15] C.E. Myers, J. Ghosh, Eur. Urol. 35 (1999) 395.

[16] G. Mamalakis, A. Kafatos, N. Kalogeropoulos, N. Andrikopoulos, G. Daskalopulos, A. Kranidis, Prostaglandins Leukot. Essent. Fatty Acids 66 (2002) 467.

[17] E. Gazi, J. Dwyer, N.P. Lockyer, P. Gardner, J. Miyan, C.A. Hart, M.D. Brown, N.W. Clarke, Biopolymers 77 (2005) 18.

[18] The European collection of cell cultures. http://www.ecacc.org.uk/ Accessed 6th January 2008.

[19] N. Abumrad, C. Coburn, A. Ibrahimi, Biochim. Biophys. Acta 1441 (1999) 4.

[20] X. Zheng, H. Baker, W.S. Hancock, F. Fawaz, M. McCaman, E. Pungor, Biotechnol. Prog. 22 (2006) 1294.

[21] L.L. Listenberger, X. Han, S.E. Lewis, C. Sylvaine, R.V. Farese, D.S. Ory, J. Schaffer, PNAS 100 (2003) 3077.

[22] Molecular Probes Handbook. A guide to Fluorescent probes for labelling technologies. Chapter 13 Probes for Lipids and Membranes. Section 13.52005 ed Spence Michelle tz. USA. Invitrogen Corp. pp. 630-631.

[23] F.H. Chilton, A.N. Fonteh, M.E. Surette, M. Triggiani, J.D. Winkler, Control of arachidonate levels within inflammatory cells, Biochim. Biophys. Acta 1299 (1996) 1-15.

[24] M. Hughes-Fulford, Y. Chen, R.R. Tjandrawinata, Fatty acid regulates gene expression and growth of human prostate cancer cells, Carcinogenesis 22 (2005) 701-707.

[25] Y. Chen, M. Hughes-Fulford, Prostaglandin E2 and the protein kinase A pathway mediate arachidonic acid induction of c-fos in human prostate cancer cells, British Journal of Cancer 82 (2000) 2000-2006. 